\title{
Management of Internal Resorption: A Catch-22 Situation
}

\author{
${ }^{1}$ Sahle AK Karanath, ${ }^{2}$ IB Geeta, ${ }^{3}$ Jayson M James
}

\begin{abstract}
Internal resorption (IR), a rare phenomenon, is considered to be a catch-22 situation from the standpoints of both its diagnosis and treatment. It begins initially in the pulpal space and extends into the surrounding dentin. Its diagnosis and management have been a challenge to dental practitioners. Due to its insidious pathology, IR can progress to a great extent before its detection. It is usually asymptomatic and discovered by chance on routine radiographic examinations or by a classic clinical sign, "pink spot" in the crown. The treatment of this condition should be initiated as soon as possible to prevent further loss of hard tissue or an eventual root perforation. The resorption, if progressed to involve an external communication, has poor prognosis, and the tooth cannot be retained in most cases, and hence, extraction of the tooth is indicated. In modern dentistry, patients demand more than restoration of function; they are particular about the esthetics as well, especially in the anterior region. Once the proper diagnosis is made, the treatment modality should include steps taken to conserve the tooth structure as much as possible to restore tooth form, function, and esthetics. This case report describes the management of an IR of a maxillary central incisor that was treated nonsurgically with conventional root canal treatment followed by reinforcing the tooth with esthetic fiber post and conserving the natural tooth as such. A 9-month follow-up demonstrated clinically asymptomatic and adequately functional tooth, with radiographic signs of healing. In this case report, care was taken to conserve the tooth structure and avoid further weakening of the already compromised tooth. A tooth deemed having questionable prognosis was preserved intact and showed satisfactory healing with no progression of the resorptive defect.
\end{abstract}

Keywords: Case report, Fiber post, Internal resorption, Pink tooth of mummery.

How to cite this article: Karanath SAK, Geeta IB, James JM. Management of Internal Resorption: A Catch-22 Situation. J Oper Dent Endod 2017;2(1):31-35.

Source of support: Nil

Conflict of interest: None

\section{INTRODUCTION}

Tooth resorption presents itself as either a physiological or a pathological process occurring internally (pulpally

\footnotetext{
${ }^{1,3}$ Postgraduate (Final Year), ${ }^{2}$ Head

1-3 Department of Conservative and Endodontics, RajaRajeswari Dental College and Hospital, Bengaluru, Karnataka, India

Corresponding Author: Sahle AK Karanath, Postgraduate (Final Year), Department of Conservative and Endodontics RajaRajeswari Dental College and Hospital, Bengaluru Karnataka, India, Phone: +919901955958, e-mail: sahak87@ gmail.com
}

derived) or externally (periodontally derived). According to the Glossary of the American Association of Endodontists, resorption is defined as a condition associated with either a physiologic or a pathologic process resulting in the loss of dentin, cementum, or bone. ${ }^{1}$

Internal resorption (IR), a rare phenomenon, is considered to be a catch-22 situation from the standpoints of both its diagnosis and treatment. It begins in the pulpal space and extends into the surrounding dentin. Its diagnosis and management have been a challenge to dental practitioners. Due to its insidious pathology, IR can progress to a great extent before its detection.

Traumatic injury, infection, and orthodontic treatment have been suggested as some of the etiological factors for IR. ${ }^{2}$ Clinically, the condition is usually asymptomatic, slowly progressing and detected by routine radiographic examination, which reveals a round-to-oval radiolucent enlargement of the pulp space on examination or by the clinical sign of a "pink spot" discoloration visible through the crown of the tooth. ${ }^{3}$ The margins are smooth and clearly defined with distortion of the original root canal outline. The treatment of this condition should be initiated as soon as possible to prevent further loss of hard tissue or an eventual root perforation.

The resorption, if progressed, to involve an external communication has poor prognosis and the tooth cannot be retained in most cases, and hence, extraction of the tooth is indicated. In modern dentistry, patients demand more than restoration of function; they are particular about the esthetics as well, especially in the anterior region. ${ }^{4}$ In addition, the psychological and economic importance of maintaining the natural tooth for the patient than replacement by osseointegrated implants should also be addressed.

In this regard, treatment modalities are included to conserve the tooth with minimal preparation as well as reinforcing the already weakened tooth and promote faster and better healing. This article describes a case of IR, which initially had a poor prognosis, but evolved favorably after conservative treatment approach and follow-up of 9 months.

\section{CASE REPORT}

A 22-year-old male patient reported to the Department of Conservative Dentistry and Endodontics with the chief complaint of pink discoloration (Fig. 1) of \#21 since 1 month. Patient gave history of orthodontic 


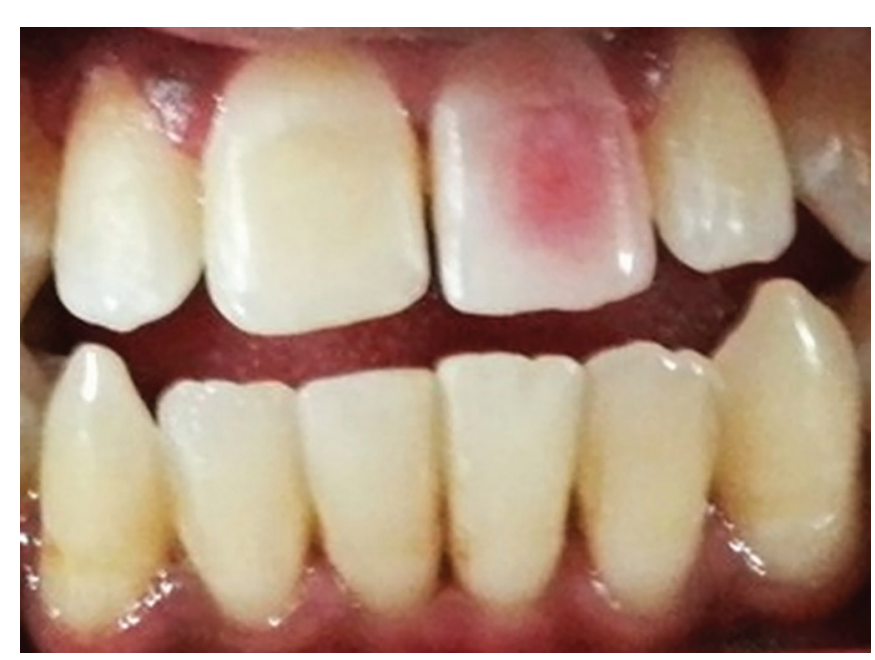

Fig. 1: Preoperative image showing pink tooth in \#21

treatment done 1 year back and had undergone endodontic treatment of \#11 with a different dentist. There was no history of any trauma as recalled by the patient. Clinical examination revealed \#21 to be slightly tender to percussion. The electric and heat pulp tests were negative for $\# 11$ and \#21. Periodontal probing depths were physiologic $(<3 \mathrm{~mm})$ at all sites. The medical history was noncontributory. Radiographic examination showed the presence of a well-defined symmetrical radiolucency at coronal and middle third of tooth, which balloons out of the root canal (Fig. 2). Based on the clinical and radiographic findings, the diagnosis was IR in \#21.

Root canal therapy to remove the necrotic pulp and disinfect the root canal system, followed by reinforcing the tooth with fiber post was recommended as the treatment modality. Endodontic therapy was initiated in \#21 after local anesthesia administration and isolation with a rubber dam. Working length determination was performed electronically using the Root ZX mini (J. Morita, USA) and confirmed radiographically. Chemomechanical preparation was done up to $60 \mathrm{~K}$ file size (Dentsply, Maillefer, USA) with copious irrigation with 3\% sodium hypochlorite. Sonic activation of irrigants (MM sonic endo 1500, MICROMEGA, France) was carried out for more efficient debridement of the canal. Canal was filled with calcium hydroxide, and closed dressing placed (Cavit G; 3M ESPE, Germany). After 2 weeks, the dressing material was removed with saline irrigation and the root canal dried with paper points. There was no bleeding from the canal, indicating that the resorption has not got any external communication. The pink discoloration of crown in \#21 had disappeared. After the master cone selection, the canal was then obturated (Fig. 3) with 2\% gutta-percha points (Dentsply, Maillefer, USA) and endoflass sealer (Sanlor laboratories, Colombia) using lateral condensation technique. The obturation was done up to the coronal third of root. The gutta-percha was then condensed using slightly heated hand plugger sized RCP 5/7 (GDC, Germany) and finger plugger sized 40

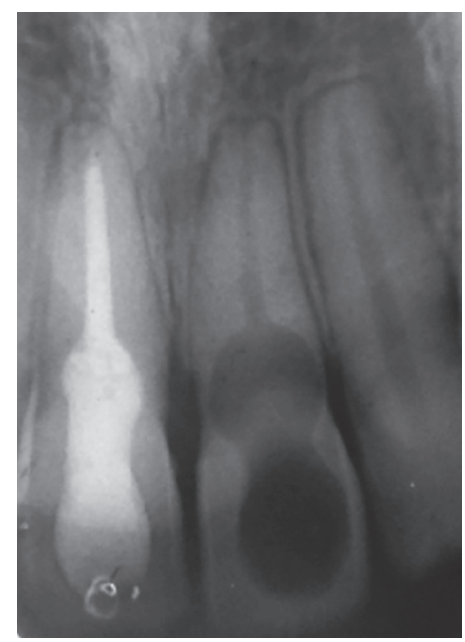

Fig. 2: Preoperative radiograph showing internal resorption in \#21

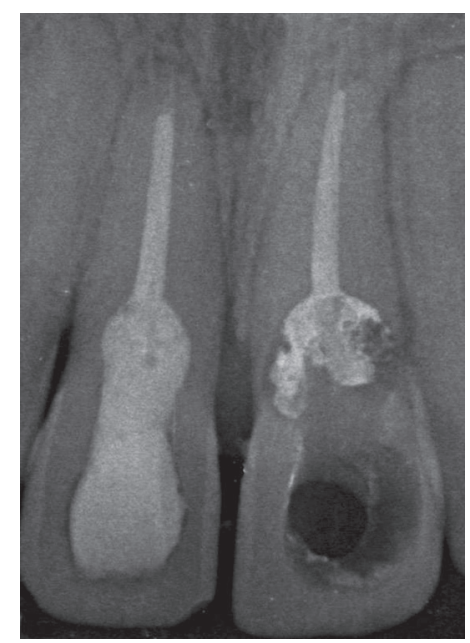

Fig. 3: Obturation

(MANI Inc, Japan) into the canal. The remaining guttapercha present in the resorptive defect was subsequently removed, while making the post space preparation and saline irrigation. The post space preparation was done using a peeso reamer of size no. 1 (MANI Inc, Japan), leaving $5 \mathrm{~mm}$ of gutta-percha at the apical third (Fig. 4). The corresponding sized fiber post (Tenax fiber transColtene, USA) was then inserted to check the fit (Fig. 5). Fiber post was luted in the post space and the entire defect was filled using dual curing core and resin cement (Paracore - dual curing core and resin cement - Coltene, USA) and light cured for 20 seconds. As the patient had open bite, it was decided not to give a prosthesis and to conserve the natural tooth as such. A radiograph taken showed the satisfactory filling of the entire resorptive defect (Fig. 6). The pink discoloration was completely eliminated following the treatment (Fig. 7). Follow-up after 9 months (Fig. 8) showed no sensitivity to percussion; periapical radiograph (Fig. 9) shows no further progression of the resorption, and no periapical changes were observed. The patient was satisfied with the treatment. 


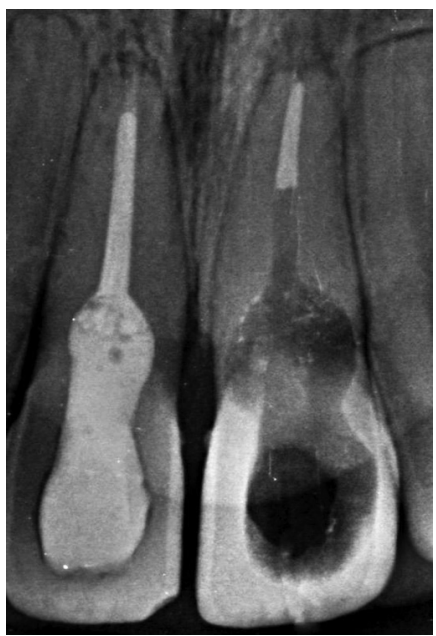

Fig. 4: Post space preparation

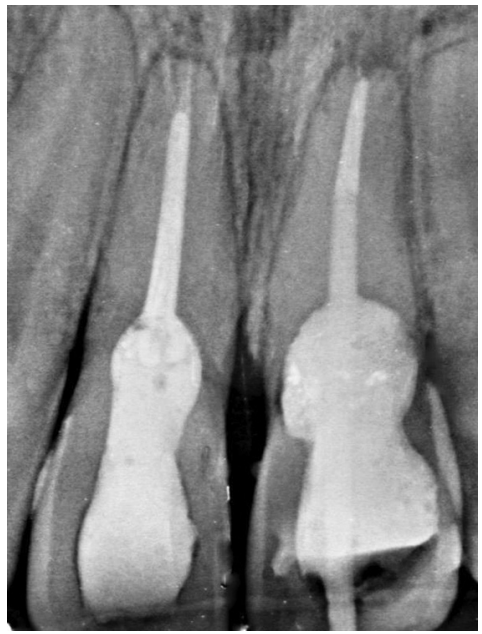

Fig. 6: Filling of entire resorptive defect

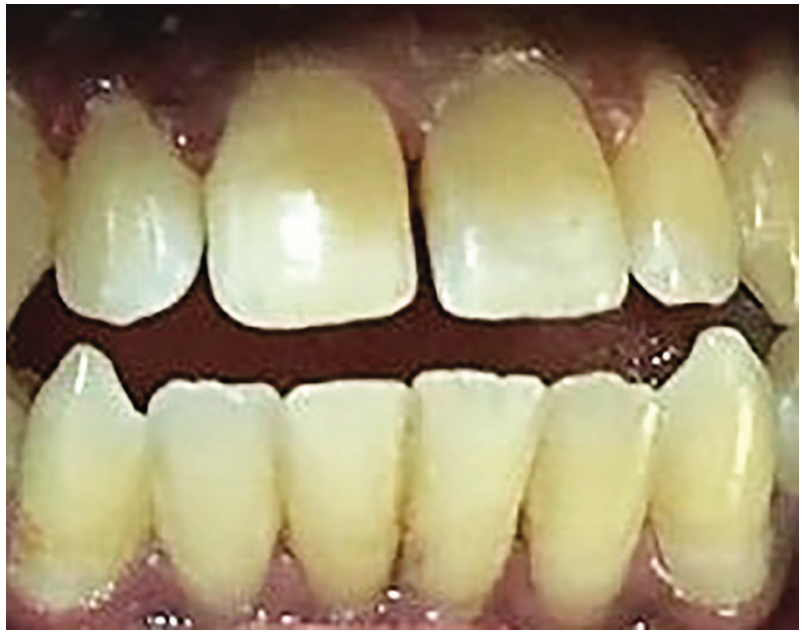

Fig. 8: Follow-up after 9 months

\section{DISCUSSION}

A tooth diagnosed with IR represents a clinical challenge to all dentists. There is always a dilemma of whether to treat a tooth with a questionable prognosis endodonti-

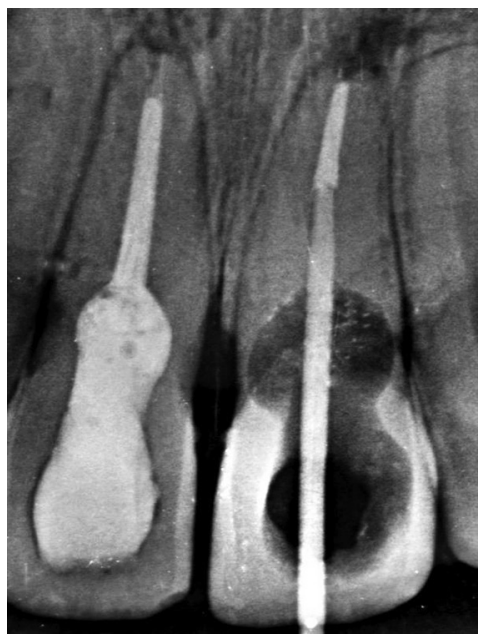

Fig. 5: Fiber post placement

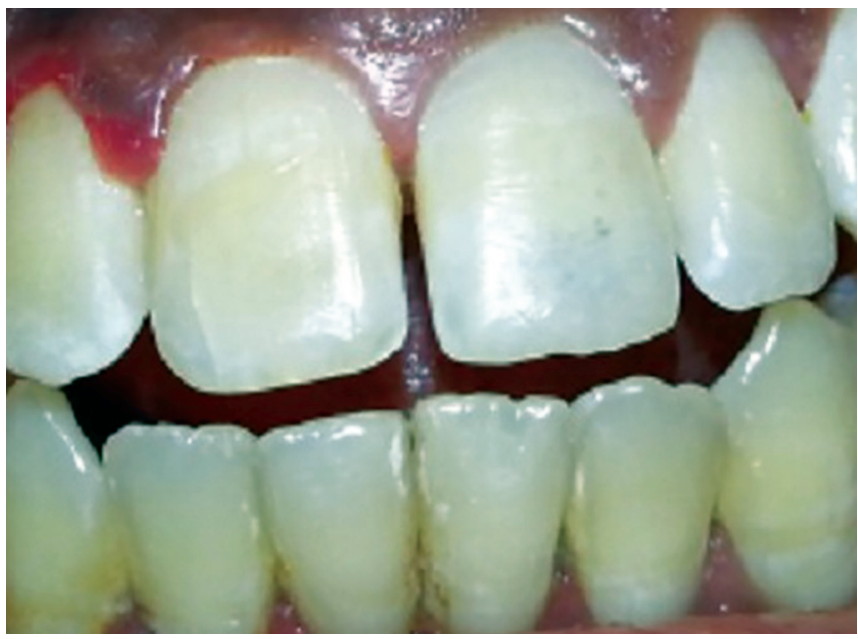

Fig. 7: Immediate postoperative

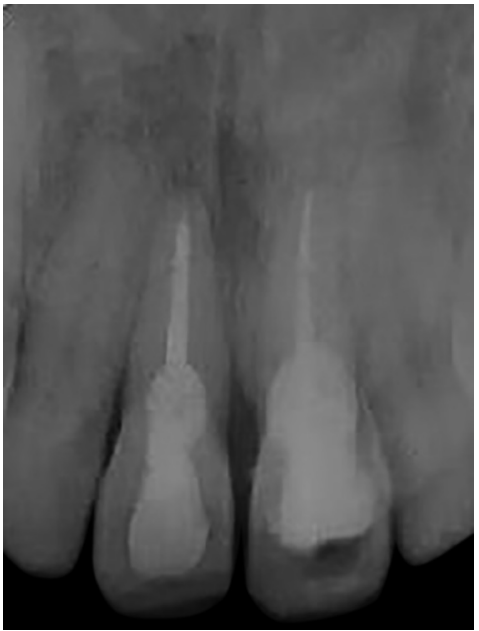

Fig. 9: Follow-up radiograph after 9 months

cally or extract it and subsequently place an implant. Tooth resorption may go unnoticed for several months or years, and there is unlikely to be any symptoms unless it is associated with significant pulpal inflammation or any 
communication with the periodontal ligaments. The IR can be detected by visual examination based on presence of a pink spot in tooth crown, radiographic diagnosis, and conventional and cone beam computed tomography. ${ }^{5}$ The patient's history will give the practitioner clues as to the possible underlying cause of IR.

In inflammatory resorption, the resorbed dentin is replaced by inflamed granulation tissue. For IR to occur, the outermost protective odontoblast layer and the predentin of the canal wall must be damaged, resulting in exposure of the underlying mineralized dentin to odontoclasts. The precise injurious events necessary to bring about such damages have not been completely elucidated. Various etiological factors have been proposed for the loss of predentin, including trauma, caries and periodontal infections, excessive heat generated during restorative procedures on vital teeth, calcium hydroxide procedures, idiopathic dystrophic changes within normal pulp, etc. ${ }^{6-12}$

In this case report, the patient's chief complaint was pink color of the crown in \#21. The pink discoloration (pink tooth of Mummery, named after the anatomist James Howard Mummery) was due to granulation tissue in growth. It is important to know if the resorption is purely internal, initiating within the pulp chamber and not communicating with the periodontal ligament. If the resorptive area is communicating, then it is an internal - external resorptive case and requires surgical management. In this case, the defect was not perforating, which was confirmed radiographically and clinically when the bleeding was controlled once the granulation tissue was completely removed.

Removal of all inflamed tissue from the resorptive defect is the basis of treatment. Hence, conventional endodontic therapy in \#21 was initiated. One of the main challenges during preparation is brisk bleeding from canal, especially when the resorption defect is active. ${ }^{3}$ Only complete effective debridement will stop the bleeding. Effective cleaning of the resorptive area with solvents having tissue-dissolving capacity is preferred. It is well known that soft tissue dissolution can be best achieved by using high concentration of sodium hypochlorite. Therefore, $3 \%$ sodium hypochlorite was used for irrigation. But sometimes complete extirpation of the inflamed tissue may become difficult by hand instruments alone. A combined approach involving both hand instrumentation and sonic activation of irrigation was performed on the involved tooth. ${ }^{13}$ Moreover, only chemomechanical debridement of the root canal space may fail to consistently render the root canal system bacteria free. Hence, an intracanal, antibacterial medicament should be used as part of disinfection process of the inaccessible areas of resorptive defects. Calcium hydroxide is antibacterial and has been shown to effectively eradicate bacteria that persist after chemomechanical instrumentation. Calcium hydroxide has also been shown to have a synergistic effect when used in conjunction with sodium hypochlorite to remove organic debris from the root canal. ${ }^{14,15}$ Placement of intracanal medication like calcium hydroxide is usually suggested for a period of 1 to 2 weeks, and because of its high alkalinity, it will inhibit clastic activity that causes resorption. ${ }^{3}$ Therefore, in the present case, calcium hydroxide dressing was given for a duration of 2 weeks. Obturation could be best achieved by warm gutta-percha techniques, either thermomechanical or thermoplasticized, to enhance the filling of resorptive defect area. But, lack of tooth structure in \#21 necessitated it to be supported with an anchorage system. Hence, esthetic light-transmitting fiber posts were used to reinforce the tooth. When such posts were bonded in place with resin cement, forces would be distributed more evenly in the root, resulting in fewer root fractures. In addition, the fiber-reinforced posts can be used with minimal preparation because resin cement uses the undercuts and surface irregularities to increase the surface area for bonding. The entire defect was filled with dual curing core and resin cement. Use of fiber posts over metal posts has advantage of monoblock effect, esthetics, and flexibility. Fiber posts also have approximately the same modulus of elasticity as dentin. ${ }^{16}$ This reduces the already weakened tooth from further possibility of fracture during function.

In the present case, care was taken to preserve as much tooth structure as possible with conservative access cavity preparation. The patient was not given a prosthesis, as he had open bite for which treatment was advised. The patient was satisfied with the treatment outcome and was recalled for periodic follow-up.

\section{CONCLUSION}

The IR, a rare phenomenon, is considered to be a catch-22 situation from the standpoint of both its diagnosis and treatment. Management of IR is a challenge to clinicians. Early diagnosis, removal of the cause, and proper treatment options in the interest of the tooth are mandatory for successful treatment outcome to prevent overweakening of remaining tooth structure and root perforations. In this case report, care was taken to preserve as much tooth structure as possible and avoid further weakening of the already compromised tooth. A tooth deemed having questionable prognosis was preserved intact and showed satisfactory healing with no progression of resorptive defect after a 9-month follow-up.

\section{CLINICAL SIGNIFICANCE}

Successful treatment requires proper diagnosis and a good history. Once IR has been diagnosed, the clinician must make a decision on the prognosis of the tooth and 
plan the treatment accordingly. Use of esthetic fiber posts is recommended for reinforcing the tooth structure in case of moderately resorbed tooth to preserve the tooth and restore its form, function, and esthetics, thereby enhancing the prognosis.

\section{REFERENCES}

1. American Association of Endodontics. Glossary of endodontic terms. Chicago (IL): AAE; 2014 [cited 2014]. Available from: www.aae.org/glossary.

2. Ne RF, Witherspoon DE, Gutmann JL. Tooth resorption. Quintessence Int 1999 Jan;30(1):9-25.

3. Haapasalo M, Endal U. Internal inflammatory root resorption: the unknown resorption of the tooth. Endod Topics $2006 \mathrm{Jul}$ 1;14(1):60-79.

4. Bakland LK. Root resorption. Dent Clin North Am 1992 Apr;36(2):491-507.

5. Patel S, Dawood A, Wilson R, Horner K, Mannocci F. The detection and management of root resorption lesions using intraoral radiography and cone beam computed tomography an in vivo investigation. Int Endod J 2009 Sep;42(9):831-838.

6. Silveira FF, Nunes E, Soares JA, Ferreira CL, Rotstein I. Double "pink tooth" associated with extensive internal root resorption after orthodontic treatment: a case report. Dent Traumatol 2009 Jun 1;25(3):e43-e47.

7. Cabrini RL, Maisto OA, Manfredi EE. Internal resorption of dentin: histopathologic control of eight cases after pulp amputation and capping with calcium hydroxide. Oral Surg Oral Med Oral Pathol 1957 Jan;10(1):90-96.

8. Rabinowitch BZ. Internal resorption. Oral Surg Oral Med Oral Pathol 1972 Feb;33(2):263-282.

9. Ashrafi $\mathrm{MH}$, Sadeghi EM. Idiopathic multiple internal resorptions: report of case. ASDC J Dent Child 1980 MayJun;47(3):196-199.

10. Brady J, Lewis DH. Internal resorption complicating orthodontic tooth movement. Br J Orthod 1984 Jul;11(3): 155-157.

11. Walton RE, Leonard LA. Cracked tooth: an etiology for "idiopathic" internal resorption?. J Endod 1986 Apr;12(4): 167-169.

12. Brooks JK. An unusual case of idiopathic internal root resorption beginning in an unerupted permanent tooth. J Endod 1986 Jul;12(7):309-310.

13. Estrela C, Bueno MR, Alencar AH, Mattar R, Valladares-Neto J, Azevedo BC, De Araújo Estrela CR. Method to evaluate inflammatory root resorption by using cone beam computed tomography. J Endod 2009 Nov;35(11):1491-1497.

14. Sjögren U, Figdor D, Spångberg L, Sundqvist G. The antimicrobial effect of calcium hydroxide as a short-term intracanal dressing. Int Endod J 1991 May;24(3):119-125.

15. Türkün M, Cengiz T. The effects of sodium hypochlorite and calcium hydroxide on tissue dissolution and root canal cleanliness. Int Endod J 1997 Sep 1;30(5):335-342.

16. Schwartz RS, Robbins JW. Post placement and restoration of endodontically treated teeth: a literature review. J Endod 2004 May;30(5):289-301. 